\title{
El concepto de "desarrollo sostenible" en la regulación del currículum de la Educación Secundaria Obligatoria en España*
}

\author{
The concept of "sustainable development" in the curriculum regulation of Secondary \\ Education in Spain
O conceito de "desenvolvimento sustentável" na regulação do currículo da Educação Secundária Obrigatória na Espanha

\author{
Jaume Sureda-Negre, Albert Catalán-Fernández, Olaya Álvarez-García, Rubén Comas-Forgas ${ }^{\mathrm{a}}$ \\ aDepartamento de Pedagogía Aplicada y Psicología de la Educación, Universidad de las Islas Baleares, España. \\ Correo electrónico: rubencomas@uib.es. Telf.: 0034971172378
}

\begin{abstract}
RESUMEN
En este artículo se analiza el tratamiento que se hace del concepto "desarrollo sostenible" en la normativa básica que regula el currículum de la Educación Secundaria Obligatoria en España. Para ello se analiza: qué conceptos relacionados con el de sostenibilidad aparecen en la normativa y con qué frecuencia lo hacen; en qué cursos y materias de esta etapa así como en qué elementos curriculares aparece la sostenibilidad; y, finalmente, el enfoque que se patrocina desde el cuerpo normativo. La metodología utilizada ha sido el análisis lexicográfico y de contenido de, por un lado, el principal decreto que regula la enseñanza secundaria obligatoria en España, el Real Decreto 1631/2006 por el que se establecen las enseñanzas mínimas correspondientes a la Educación Secundaria Obligatoria y, por otro lado, los decretos reguladores de la enseñanza secundaria obligatoria de las Comunidades Autónomas de las Islas Baleares (Decreto 73/2008) y Madrid (Decreto 23/2007).
\end{abstract}

Palabras clave: desarrollo sostenible, educación ambiental, educación secundaria obligatoria, legislación educativa.

\begin{abstract}
This article discusses the treatment of the concept "sustainable development" within the basic rules governing the curriculum of Secondary Education in Spain. The aspects in discussion are: what are the concepts related to sustainability which appear in the regulations, and how often do they appear; in which courses and subjects of this school stage, as well as in which curriculum elements are they present; and finally, the approach sponsored from the regulatory body. The methodology used was the lexical and content analysis of: on the one hand, the main decree regulating compulsory secondary education in Spain; the Royal Decree 1631/2006 establishing the core curriculum for compulsory secondary education; and on the other hand, the regulating decrees for compulsory secondary education in the Autonomous Communities of the Balearic Islands (Decree 73/2008) and Madrid (Decree 23/2007).
\end{abstract}

Key words: sustainable development, environmental education, compulsory secondary education, educational legislation.

\section{RESUMO}

Analisa-se o tratamento dado ao conceito de "desenvolvimento sustentável" no conjunto de normas básicas que regem o currículo da Educação Secundária Obrigatória na Espanha. Para isso, analisa-se a frequência e quais conceitos relacionados à sustentabilidade aparecem no referido conjunto de normas; em quais cursos e disciplinas desta etapa e em quais conteúdos curriculares a sustentabilidade aparece além do enfoque que se patrocina desde o corpo normativo. Utilizou-se a metodologia da análise lexicográfica e de conteúdo de, por um lado, o principal decreto que regulamenta a Educação Secundária Obrigatória na Espanha, o Real Decreto 1631/2006, que estabelece o ensino mínimo correspondentes ao citado nível de ensino e, por outro, os decretos que regulam a Educação Secundária Obrigatória nas Comunidades Autônomas das Ilhas Baleares (Decreto 73/2008) e Madrid (Decreto 23/2007).

Palavras chave: desenvolvimento sustentável, educação ambiental, educação secundária obrigatória, legislação educacional. 


\section{INTRODUCCIÓN}

Aunque el concepto fue utilizado ya en los años setenta, es a partir de 1987, con la publicación del Brundtland Commission Report, y sobre todo de los acuerdos adoptados en la conferencia de Río de Janeiro sobre medio ambiente y desarrollo, en 1992, cuando la idea de "desarrollo sostenible" se populariza y cobra importancia, no sólo en los debates centrados en el medio ambiente sino también en otros muchos ámbitos. Así, en el campo educativo, la Asamblea General de las Naciones Unidas, mediante la resolución 57/254, aprobada el 20 de Diciembre de 2002, proclamó la Década de la educación para el Desarrollo Sostenible (2005-2014), donde se pone de manifiesto que "la educación es un elemento indispensable para alcanzar el desarrollo sostenible". Indica además, que la Educación para el Desarrollo Sostenible "pretende proporcionar a toda persona la oportunidad de adquirir los valores, competencias, conocimientos y habilidades que le permitan contribuir a un futuro humano justo, económicamente viable y ecológicamente sostenible" (UNESCO, 2009). En la misma línea, Tilbury y Wortman (2004) señalan que la educación es fundamental para promover el desarrollo sostenible y Vare y Scott (2008: 192) sostienen que "hoy en día, cada nivel del sistema educativo es instado a declarar su respaldo a la educación para el desarrollo sostenible". Naturalmente, la cuestión inmediata que se plantea se refiere a las demandas y consecuencias que puede tener para el sistema educativo, el español en este caso, la aceptación de su papel crucial en el camino hacia la sostenibilidad, sobre todo considerada la relativa -tal vez no inocente- ambigüedad del concepto (Fergus y Rowney, 2005) y los requerimientos de toda índole necesarios para su presencia sustantiva en el proceso educativo.

A pesar de la gran importancia que se otorga al concepto, no sólo hay diversas formas de interpretarlo -a partir de la definición inicial de la Comisión Brundtland se han propuesto numerosas caracterizaciones- sino que además hay múltiples evidencias que demuestran que la comprensión que diversos colectivos tienen del mismo es diversa, parcial y limitada, condicionada a menudo por los valores e intereses propios de los mismos. Se ha dicho, y no sin razón, que el desarrollo sostenible es una de estas ideas que todo el mundo apoya pero de la que nadie conoce su significado ${ }^{1}$ o de la que se tiene una visión muy limitada. De ello dan fe numerosos trabajos empíricos centrados en analizar qué entienden por desarrollo sostenible diversos colectivos, por ejemplo: menores de edad (Rodríguez, Kohen y Delvao, 2008; Walshe, 2008); estudiantes universitarios (Aragonés, Izurieta y Raposo 2000, 2003; Moreira y Carneiro, 2007; Reid, Petocz y Taylor, 2009); profesores universitarios (Cotton, Warren, Maiboroda y Bailey, 2007); profesorado en formación o en activo (Summers, Corney y Chile, 2004; Corney y Reid, 2007; Firth y Winter 2007; Corney 2006; Winter y Firth 2007; Ärlemalm-Hagser y Sandberg 2011); líderes de opinión (Byrch, Kearins, Milne y Morgan, 2007). Sea como fuere, esta falta de precisión, hasta cierto punto, no la podemos considerar negativamente dado que, tal y como sostienen Robinson (2004), Kemp y Martens (2007) y Jóhannesson et al. (2011), una conceptualización cerrada y unívoca del término excluiría puntos de vista e interés

"Sustainable development is one of those ideas that everybody supports but nobody knows what it means" (Porritt citado en Financial Times, 1998). 
alejados de la definición estandarizada y de esta manera restaría el carácter de "paradigma para el pensamiento" (Jóhannesson et al., 2011) y se acercaría a un estado de dogma que poco o nada tiene que ver con el concepto en sí.

Ya hemos señalado que la idea de desarrollo sostenible también se ha introducido en el campo educativo, entre otras razones a partir de la necesidad de educar a las jóvenes generaciones para un futuro mejor. Cabe preguntarse, por tanto, cómo contribuye el sistema escolar a promocionar la concepción del desarrollo sostenible y la acción a favor del mismo. En este artículo planteamos la cuestión de cómo la legislación general que regula la Educación Secundaria Obligatoria (ESO) en España trata el tema de la sostenibilidad,y si promociona una determinada forma de entenderla. ${ }^{2}$ Esta tarea y la manera como la abordamos implica la asunción de ciertas limitaciones: somos conscientes de que un análisis exhaustivo de cómo el concepto "desarrollo sostenible" y afines es contemplado en la escuela, implicaría analizar por lo menos: (a) las normativas que regulan el desarrollo curricular; (b) los diversos documentos de concreción curricular (programaciones de centro y programaciones de aula); (c) los recursos y medios didácticos utilizados (entre ellos, singularmente, los libros de texto); (d) la organización y dinámica de las aulas; (e) la forma en la que el concepto es trabajado, entendido y asumido por el profesorado y el alumnado; f) la forma en que se evalúa la adquisición de los diversos contenidos relacionados con la sostenibilidad y el desarrollo de las competencias necesarias para su aplicación a situaciones o problemas concretos.

Nuestro trabajo se limita al primero de los puntos señalados, al análisis de la normativa legal básica en la que se enmarca la acción educativa. La propuesta que aquí se presenta se enmarca en la línea de investigaciones de similares características como son las aportaciones de Jóhannesson et al. (2011), quienes analizaron el tratamiento que se da en el currículum educativo islandés al concepto de desarrollo sostenible. Su trabajo se basó en el análisis de más de 100 documentos curriculares de los niveles educativos islandeses que van desde la etapa preescolar (entre 1 y 6 años), pasando por la etapa intermedia u obligatoria (entre 6 y 16 años), hasta alcanzar la etapa pre-universitaria o upper-secondary education (entre 16 y 20 años de edad), utilizando para ello una clave basada en siete características indicativas de una educación para el desarrollo sostenible. ${ }^{3}$ Similares características, aunque limitado al análisis del concepto desarrollo sostenible en el currículum de Tecnología en la educación secundaria irlandesa, presenta el trabajo de McGarr (2010). Por último, conviene citar la labor de Rëihnlander y Kramer (2003) quienes, centrados en analizar los niveles superiores del sistema educativo, describieron la incorporación del concepto de sostenibilidad en el currículum de las carreras de economía y administración de la empresa en Alemania.

$\overline{2} \quad$ Winter (2007) ya puso de manifiesto la manera confusa con que la educación para el desarrollo sostenible es presentada en diversos currículos y normativas, provocando ello una inexistencia de guías y referentes claros para los docentes.

3 1) Referencias a valores, opiniones y sentimientos acerca de la naturaleza y el medio ambiente; 2) Identificación de conocimientos que pueden contribuir a un uso sensato de la naturaleza; 3) Declaraciones sobre el bienestar y la salud pública; 4) Referencias a competencia para la democracia, la participación y la acción; 5) Reconocimiento de la igualdad y multiculturalidad; 6) Referencias a la conciencia y la comprensión de temas globales; y 7) Referencias al desarrollo económico y las perspectivas de futuro. (Jóhannesson et al, 2011) 
Finalmente, para delimitar e identificar la etapa educativa sobre la que trata este artículo, resulta pertinente señalar que el actual sistema educativo español está regulado por la LOE (Ley Orgánica 2/2006, de 3 de mayo) en la que se señalan, entre otras cosas, los principios y fines de la educación así como los ciclos y etapas. Su artículo 6.2 fija que es competencia del Gobierno del Estado fijar las enseñanzas mínimas (aspectos básicos de sus objetivos, competencias básicas, contenidos y criterios de evaluación) para el conjunto de España, dejando un considerable nivel de decisión a las diversas comunidades autónomas (en total son 17 ) en que está estructurado el Estado español. Los aspectos básicos (las enseñanzas mínimas) del currículum de secundaria para el conjunto de España se establecieron a través del Real Decreto 1631/2006 de 29 de diciembre, por el que se establecen las enseñanzas mínimas correspondientes a la ESO. Posteriormente, y a partir de las orientaciones de este real decreto, los gobiernos de las comunidades autónomas concretaron el currículo de dicha enseñanza para los centros de su territorio respectivo. En este trabajo nos hemos centrado en el análisis del decreto general para toda España y de los de decretos reguladores de Madrid (Decreto 23/2007) y las Islas Baleares (73/2008) (véase Esquema 1). Decidimos no ampliar el estudio a los decretos de la totalidad de comunidades al comprobar a través de un análisis lexicográfico que en los decretos regionales se utilizaban no solo los mismos conceptos relacionados con la sostenibilidad sino también en porcentajes casi idénticos.

\section{OBJETIVOS}

El objetivo de este trabajo consiste en analizar la idea de desarrollo sostenible en la normativa que regula la enseñanza secundaria obligatoria en España y para ello se responde a las cuestiones siguientes:

a) ¿Qué conceptos directamente relacionados con la sostenibilidad aparecen en los decretos que regulan el currículum de la ESO? ¿Con qué frecuencia aparecen? ¿Qué importancia se da a estos conceptos?

b) ¿En qué disciplinas y en qué cursos establecen estos decretos la enseñanza de la sostenibilidad? ¿Qué contenidos se proponen?

c) ¿Qué enfoque / paradigma de la sostenibilidad se adopta en los decretos? ¿Se propone un mismo enfoque en todas las disciplinas?

\section{METODOLOGÍA}

El método utilizado para responder a las cuestiones planteadas ha consistido en el análisis lexicográfico y de contenido del Real Decreto 1631/2006 y, para ampliar el foco del análisis, también se han analizado los decretos de las comunidades autónomas de Madrid y de las Islas Baleares. Como se ha indicado, para discernir el enfoque del desarrollo sostenible implícito en la normativa que regula el currículum de la educación secundaria en España se han analizado todas aquellas referencias al desarrollo sostenible incluidas en el Real decreto, de aplicación en todo el país. De cada texto con referencias a la sostenibilidad se han considerado todas aquellas oraciones con sentido lógico que reflejan una determinada caracterización de la misma. 
A partir del análisis de todas las oraciones se establecieron diversas dimensiones nodos- que permiten clasificar las referencias a la sostenibilidad incluidas en los decretos analizados. Este proceso de análisis se realizó de forma independiente por dos de los autores de este trabajo. Para el tratamiento de la información hemos utilizado el programa NVIVO.

\section{RESULTADOS}

\subsection{LOS CONCEPTOS DE SOSTENIBILIDAD (CAMPO SEMÁNTICO) EN LA NORMATIVA ANALIZADA}

El análisis lexicográfico muestra que en el decreto que regula el currículum de la ESO para el conjunto de España, la palabra sostenible, ya sea como sustantivo o como adjetivo, aparece en siete formas o expresiones diferentes: 1) Desarrollo sostenible (DS); 2) Sostenibilidad (S); 3) Desarrollo humano sostenible (DHS); 4) Gestión sostenible (GS); 5) Futuro sostenible (FS); 6) Aprovechamiento sostenible (AS); y 7) Sociedades democráticas sostenibles (SDS). Estos conceptos aparecen en 24 ocasiones en total. Estos mismos términos son los que aparecen también en la normativa de la Comunidad de Madrid y en la de las Islas Baleares; en estos casos, sin embargo, aparecen en 17 y en 31 ocasiones respectivamente (véase Esquema 1). A pesar de estas diferencias, es destacable el hecho de que los porcentajes de aparición de estos conceptos son prácticamente idénticos.

Esquema 1. Número de ocasiones en los que aparece el concepto desarrollo sostenible -y otros vocablos de su mismo campo semántico- en las principales normas que regulan la ESO en el conjunto de España y en las Comunidades Autónomas de Madrid y Baleares.

\begin{tabular}{|c|c|c|c|c|c|c|c|c|}
\hline & $\begin{array}{c}\text { Desarrollo } \\
\text { sostenible }\end{array}$ & Sostenibilidad & $\begin{array}{c}\text { Desarrollo } \\
\text { humano } \\
\text { sostenible }\end{array}$ & $\begin{array}{c}\text { Gestión } \\
\text { sostenible }\end{array}$ & $\begin{array}{c}\text { Futuro } \\
\text { sostenible }\end{array}$ & $\begin{array}{c}\text { Aprovechamiento } \\
\text { sostenible }\end{array}$ & $\begin{array}{c}\text { Sociedades } \\
\text { democráticas } \\
\text { sostenibles }\end{array}$ & $\begin{array}{c}\text { Número } \\
\text { total de } \\
\text { referencias }\end{array}$ \\
\hline $\begin{array}{c}\text { Real } \\
\text { Decreto } \\
1631 / 2006\end{array}$ & 7 & 5 & 3 & 3 & 4 & 1 & 1 & 24 \\
\hline $\begin{array}{c}\text { Decreto } \\
73 / 2008 \\
\text { Baleares }\end{array}$ & 10 & 8 & 2 & 5 & 3 & 2 & 1 & 31 \\
\hline $\begin{array}{c}\text { Decreto } \\
23 / 2007 \\
\text { Madrid }\end{array}$ & 7 & 4 & 1 & 1 & 3 & 0 & 1 & 17 \\
\hline $\begin{array}{c}\text { Número } \\
\text { total }\end{array}$ & 24 & 17 & 6 & 9 & 10 & 3 & 3 & 72 \\
\hline
\end{tabular}

\subsection{LA SOSTENIBILIDAD Y LAS MATERIAS Y ASIGNATURAS DEL CURRÍCULUM DE ESO}

En España, la ESO está estructurada en cuatro cursos que se siguen ordinariamente entre los doce y los dieciséis años de edad. En los tres primeros se imparten las siguientes materias: Ciencias de la naturaleza, que en tercero puede desdoblarse en Biología y geología, por un lado, y Física y química por otro; Ciencias sociales, geografía e historia; Educación física; Lengua castellana y literatura y, si la hubiere, lengua cooficial y literatura.; 
Lengua extranjera; Matemáticas; Educación plástica y visual; Música y Tecnologías, aunque estas tres últimas se imparten solamente en dos de los tres primeros cursos. Además, en uno de estos tres primeros cursos debe cursarse la asignatura de Educación para la ciudadanía y los derechos humanos. En el cuarto curso todos los alumnos deben cursar seis materias obligatorias, siete en las comunidades con lengua cooficial (Ciencias sociales, geografía e historia; Educación ético-cívica; Educación física; Lengua castellana y literatura y, si la hubiere, lengua cooficial y literatura; Matemáticas y Primera lengua extranjera) y tres a escoger entre estas ocho: Biología y geología; Educación plástica y visual; Física y química; Informática; Latín; Música; Segunda lengua extranjera y, finalmente, Tecnología). La ESO es la última etapa obligatoria del sistema educativo español.

El esquema 2 recoge las referencias al desarrollo sostenible y conceptos relacionados que aparecen en el Real Decreto 1631/2006 y en los correspondientes decretos que lo desarrollan en las comunidades autónomas de las Islas Baleares y Madrid, respectivamente. Consideradas conjuntamente estas tres disposiciones legales, el mayor número de referencias se hallan en el área de Ciencias de la Naturaleza (38) y dentro de ésta en la asignatura de Física y química de $4^{\circ}$ curso (25), repartiéndose el resto entre los otros tres cursos. A continuación se sitúa el área de Ciencias sociales, Geografía e Historia, con 12 referencias en total, destacando las que corresponden a $3^{\circ}$, con 8 referencias. El resto de áreas que presentan referencias a la sostenibilidad son las Tecnologías (7); Educación para la ciudadanía y los derechos humanos (5); Educación plástica y visual (3), e Historia y cultura de las religiones (1, solamente en Baleares)

Esquema 2. Número de ocasiones en los que aparece el concepto desarrollo sostenible -y otros vocablos de su mismo campo semántico- en las diversas materias que configuran el contenido de la ESO

\begin{tabular}{|c|c|c|c|}
\hline Materias & Real Decreto & Decreto de Baleares & Decreto de Madrid \\
\hline $\begin{array}{c}\text { Ciencias de } \\
\text { la naturaleza. } \\
\text { (Número total de } \\
\text { referencias: } 38 \text { ) }\end{array}$ & $\begin{array}{c}\text { Número total de referencias: } 15 \\
\text { - Contenidos asignatura Física } \\
\text { y química de cuarto curso (5 } \\
\text { referencias) } \\
\text { - Criterios evaluación Física } \\
\text { y química de cuarto curso (3 } \\
\text { referencias) } \\
\text { - Introducción a la materia. Física } \\
\text { y química de cuarto curso } \\
\text { • Contribución materia a } \\
\text { adquisición competencias básicas. } \\
\text { Física y química de cuarto curso } \\
\text { - Criterios evaluación primer curso } \\
\text { - Contenidos tercer curso Biología } \\
\text { y geología } \\
\text { - Objetivos de la materia } \\
\text { - Criterios evaluación segundo } \\
\text { curso } \\
\text { - Criterios evaluación Biología y } \\
\text { Geología cuarto curso }\end{array}$ & $\begin{array}{c}\text { - Número total de referencias: } 17 \\
\text { - Contenidos Física y química } 4 \text { curso } \\
\text { (4 referencias) } \\
\text { - Criterios evaluación Física y Química } \\
4 \text { curso ( } 3 \text { referencias) } \\
\text { - Objetivos de la materia. Física y } \\
\text { química de cuarto curso ( } 2 \text { referencias) } \\
\text { - Contribución materia a adquisición } \\
\text { competencias básicas. Física y química } \\
\text { de cuarto curso (1 referencia) } \\
\text { - Contenidos primer curso (2 } \\
\text { referencias) } \\
\text { - Criterios evaluación primer curso (1 } \\
\text { referencia) } \\
\text { - Contenidos Biología y Geología } \\
\text { tercer curso (1 referencia) } \\
\text { - Criterios de evaluación Biología y } \\
\text { geología tercer curso (1 referencia) } \\
\text { - Criterios evaluación segundo curso (1 } \\
\text { referencia) } \\
\text { Criterios evaluación } 4 \text { curso Biología y } \\
\text { geología (1 referencia) }\end{array}$ & $\begin{array}{l}\text { - Número total de } \\
\text { referencias: } 7 \\
\text { - Contenidos Física } \\
\text { y química } 4 \text { curso: } \\
\text { (4 referencias:) } \\
\text { - Contribución } \\
\text { materia a } \\
\text { adquisición } \\
\text { competencias } \\
\text { básicas } \\
\text { - Contenidos tercer } \\
\text { curso Biología y } \\
\text { Geología } \\
\text { - Objetivos } \\
\text { generales de la } \\
\text { materia }\end{array}$ \\
\hline
\end{tabular}




\begin{tabular}{|c|c|c|c|}
\hline $\begin{array}{l}\text { Ciencias sociales, } \\
\text { geografía } \\
\text { e historia. } \\
\text { (Número total de } \\
\text { referencias: } 13 \text { ) }\end{array}$ & $\begin{array}{c}\text { Número total: } 5 \\
\text { - Contenidos primer curso } \\
\text { - Contenidos tercer curso Ciencias } \\
\text { sociales } \\
\text { - Criterios evaluación tercer curso } \\
(2 \text { referencias }) \\
\text { - Contenidos tercer curso }\end{array}$ & $\begin{array}{c}\text { Número total: } 5 \\
\bullet \text { Introducción } \\
\bullet \text { Contenidos primer curso } \\
\cdot \text { Criterios evaluación primer curso } \\
\cdot \text { Contenidos tercer curso } \\
\cdot \text { Criterios evaluación tercer curso }\end{array}$ & $\begin{array}{l}\text { Número total: } 3 \\
\text { - Contenidos tercer } \\
\text { curso Geografía } \\
\text { - Contenidos primer } \\
\text { curso Geografía } \\
\text { - Contenidos tercer } \\
\text { curso Geografía }\end{array}$ \\
\hline $\begin{array}{l}\text { Educación } \\
\text { plástica y visual. } \\
\text { (Número total de } \\
\text { referencias: } 3 \text { ) }\end{array}$ & $\begin{array}{c}\text { Número total: } 1 \\
\text { - Introducción a la materia }\end{array}$ & $\begin{array}{c}\text { Número total: } 1 \\
\text { - Contribución de la materia a la } \\
\text { adquisición competencias básicas }\end{array}$ & $\begin{array}{l}\text { Número total: } 1 \\
\text { - Introducción } \\
\text { materia }\end{array}$ \\
\hline $\begin{array}{l}\text { Tecnologías. } \\
\text { (Número total de } \\
\text { referencias; 7) }\end{array}$ & $\begin{array}{c}\text { Número total: } 1 \\
\text { - Contenidos cuarto curso }\end{array}$ & $\begin{array}{c}\text { Número total: } 4 . \\
\text { - Contenidos tercer curso } \\
\text { • Criterios evaluación tercer curso } \\
\text { - Contenidos cuarto curso }\end{array}$ & $\begin{array}{c}\text { Número total: } 3 \\
\text { • Criterios } \\
\text { evaluación tercer } \\
\text { curso } \\
\text { • Contenidos tercer } \\
\text { curso } \\
\text { • Criterios } \\
\text { evaluación tercer } \\
\text { curso } \\
\text { • Contenidos cuarto } \\
\text { curso } \\
\text { • Criterios } \\
\text { evaluación cuarto } \\
\text { curso }\end{array}$ \\
\hline $\begin{array}{l}\text { Educación para } \\
\text { la ciudadanía } \\
\text { y los derechos } \\
\text { humanos. } \\
\text { (Número total de } \\
\text { referencias: 5) } \\
\end{array}$ & $\begin{array}{l}\text { Número total: } 2 \\
\text { - Introducción a la materia } \\
\text { - Contenidos } 4 \text { curso }\end{array}$ & $\begin{array}{l}\text { Número total: } 2 \\
\text { - Introducción haciendo referencia a } \\
\text { contenidos } \\
\bullet \text { Contenidos }\end{array}$ & $\begin{array}{l}\text { Número total: } 1 \\
\text { • Contenidos } \\
\text { educación ético- } \\
\text { cívica } 4 \text { curso }\end{array}$ \\
\hline $\begin{array}{l}\text { Historia y cultura } \\
\text { de las religiones. } \\
\text { (Número de } \\
\text { referencias; } 1 \text { ) }\end{array}$ & & $\begin{array}{l}\text { Número total: } 1 \\
\text { • Contribución de la materia a la } \\
\text { adquisición de competencias básicas }\end{array}$ & \\
\hline
\end{tabular}

\subsection{EL ENFOQUE DE LA SOSTENIBILIDAD}

Para determinar qué concepto de sostenibilidad se patrocina desde la norma que regula el currículum de la ESO para el conjunto de España hemos seleccionado y analizado todas aquellas frases referidas a la sostenibilidad que aparecen en el decreto regulador -en total son 59 frases- y las hemos clasificado en siete categorías o dimensiones:

- Desarrollo científico-tecnológico: en esta categoría incluimos aquellas referencias a la contribución del desarrollo tecnocientífico a la consecución de la sostenibilidad, a la resolución de los problemas (DCT)

- Problemas ambientales y/o sociales: referencias a problemas ambientales o sociales para cuya solución se hace referencia a la sostenibilidad o que aparecen asociados a este concepto (PAS) 
- Comportamientos (y actitudes) individuales y colectivos: referencias a la necesidad de actuar para avanzar hacia la sostenibilidad. Esta actuación puede ser genérica o hacer referencia a: 1) Comportamientos individuales: referencias que asocian comportamientos individuales con la sostenibilidad; 2) Decisiones colectivas y participación ciudadana: referencias que asocian la sostenibilidad con decisiones y actuaciones colectivas (C)

- Interdependencia, globalización: referencias que relacionan la sostenibilidad con la interdependencia y la globalización (IG)

- Principio de precaución: referencias a este principio relacionado con la sostenibilidad (PP)

- Ética: referencias a la importancia de la perspectiva ética en el análisis de la problemática ambiental (ET)

- Economía: referencias a los efectos medioambientales de las actividades económicas (ECO)

De este modo, disponemos de un instrumento para el análisis básico del currículum, que puede aplicarse de modo relativamente sencillo, identificando aquellas frases en las que, como mínimo, aparece asociado uno de los conceptos del campo semántico de la sostenibilidad (que incluye siete conceptos, según se recoge en el Esquema 1, en el estudio realizado a partir del Real Decreto), y una de las siete dimensiones o categorías que la caracterizan, establecidas también a partir de dicho estudio).

En el esquema 3 se clasifican las referencias a la sostenibilidad de acuerdo a estas categorías. Cabe señalar que esta tarea no ha resultado fácil, ya que la redacción de las referencias y su misma relación con la categoría o dimensión correspondiente no siempre ofrece una interpretación evidente. Este aspecto, aparte de la dificultad metodológica que presenta al objetivo de este trabajo, ofrece también otro aspecto a la reflexión, ya que en definitiva serán los propios docentes quienes deberán dar sentido al texto legal y aplicarlo a la práctica educativa. La ambigüedad en los significados puede resultar un inconveniente, pero también, sin duda, una ventaja para aquellos docentes dispuestos a desarrollar un programa ambicioso de educación ambiental para la sostenibilidad.

Conviene recordar también, una vez más, que el texto legal estudiado recoge otras referencias inequívocas relacionadas con la sostenibilidad (problemas ambientales, visión sistémica, etc.). En el presente trabajo, con el fin de ajustarse a su finalidad general, se ha adoptado una metodología en la que se recogen únicamente las referencias que aparecen incluidas en los textos que recogen simultáneamente, y como mínimo, uno de los conceptos del campo semántico de la sostenibilidad y una de las categorías o dimensiones con las que aquella aparece asociada. Hechas, pues, las aclaraciones precedentes, se expone a continuación el Esquema 3 y los resultados resumidos, obtenidos a partir del mismo. 
Esquema 3. Referencias vinculadas a las diversas dimensiones de la sostenibilidad que aparecen en el RD 1631/2006

\begin{tabular}{|c|c|}
\hline $\begin{array}{l}\text { DCT (importancia del } \\
\text { desarrollo científico- } \\
\text { tecnológico) }\end{array}$ & $\begin{array}{c}\text { • Desarrollo tecnocientífico para la sostenibilidad } \\
\text { • Contribución del desarrollo tecnocientífico a la resolución de los } \\
\text { problemas } \\
\text { - Interacciones de la ciencia y la tecnología con la sociedad y el medio } \\
\text { ambiente } \\
\text { - Responsabilidad de la ciencia y la tecnología y la necesidad de su } \\
\text { implicación para resolverlos } \\
\text { - Valoración del desarrollo tecnológico a lo largo de la historia } \\
\text { • Las implicaciones que la actividad humana y, en particular, } \\
\text { determinados hábitos sociales y la actividad científica y tecnológica } \\
\text { tienen en el medio ambiente } \\
\text { - Evitar caer en actitudes simplistas de exaltación o de rechazo del papel } \\
\text { de la tecnociencia }\end{array}$ \\
\hline $\begin{array}{c}\text { PAS } \\
\text { (problemas ambientales } \\
\text { y/o sociales (agua, energía, } \\
\text { etc.)) }\end{array}$ & 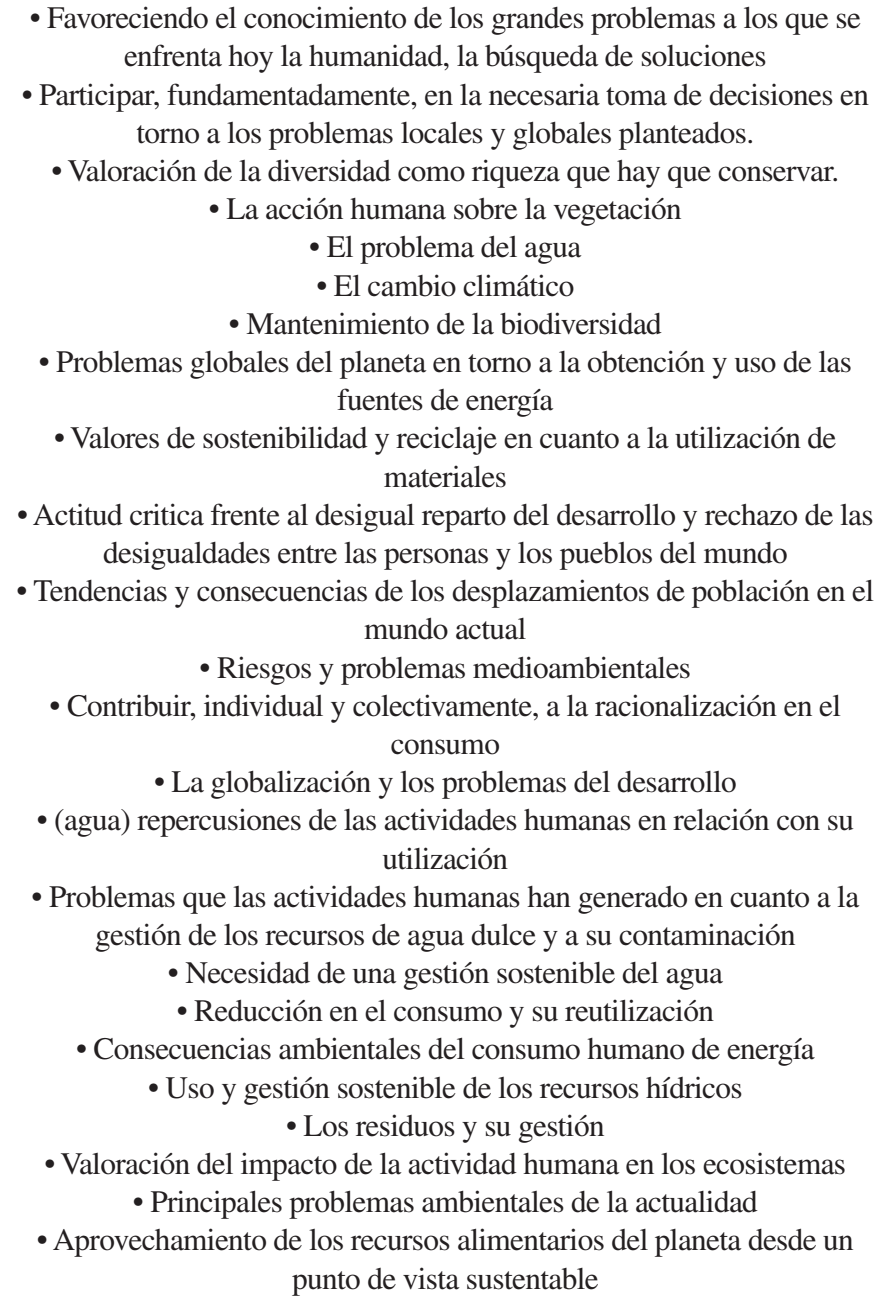 \\
\hline
\end{tabular}




\begin{tabular}{|c|c|}
\hline & $\begin{array}{l}\text { - Repercusiones de las actividades humanas en el mantenimiento de la } \\
\text { biodiversidad en los ecosistemas } \\
\text { - Reconocer la importancia y repercusiones para la sociedad y el medio } \\
\text { ambiente de las diferentes fuentes de energía renovables y no renovables } \\
\text { - (Fuentes de energía renovables y no renovables) Problemas asociados a } \\
\text { su obtención, transporte y utilización } \\
\text { - Importancia del ahorro energético y el uso de energías limpias } \\
\text { - Problemas y desafíos, estrechamente relacionados, a los que se enfrenta } \\
\text { la humanidad en relación con la situación de la tierra } \\
\text { - Los problemas y desafíos globales a los que se enfrenta hoy la } \\
\text { humanidad } \\
\text { - Contaminación sin fronteras } \\
\bullet \text { Cambio climático } \\
\text { •Agotamiento de recursos } \\
\text { • Pérdida de biodiversidad }\end{array}$ \\
\hline $\begin{array}{l}\text { C (comportamientos } \\
\text { individuales y colectivos; } \\
\text { participación...) }\end{array}$ & $\begin{array}{l}\text { - Facilitando actitudes responsables dirigidas a sentar las bases de un } \\
\text { desarrollo sostenible } \\
\text { - La necesidad de actuar para avanzar hacia el logro de un desarrollo } \\
\text { sostenible } \\
\text { • Las implicaciones que la actividad humana y, en particular, } \\
\text { determinados hábitos sociales (...) Tienen en el medio ambiente } \\
\text { • La formación básica para participar, fundamentadamente, en la } \\
\text { necesaria toma de decisiones en torno a los problemas locales y globales } \\
\text { planteados } \\
\text { • Disposición favorable para contribuir al mantenimiento de la } \\
\text { biodiversidad y a un desarrollo sostenible } \\
\text { - Describir algún caso que muestre las consecuencias medioambientales } \\
\text { de (...) Los comportamientos individuales } \\
\text { - Aportando algún ejemplo de los acuerdos y políticas internacionales } \\
\text { para frenar su deterioro } \\
\text { - Sugiriendo actuaciones y políticas concretas que mejoran la calidad } \\
\text { ambiental y colaboran en la búsqueda de un desarrollo sostenible } \\
\text { • Adquisición de hábitos que potencien el desarrollo sostenible } \\
\text { • Disposición favorable para contribuir, individual y colectivamente, } \\
\text { a la racionalización en el consumo y al desarrollo humano de forma } \\
\text { equitativa y sostenible } \\
\text { - Importancia (...) De la participación ciudadana en la toma de decisiones }\end{array}$ \\
\hline $\begin{array}{l}\text { IG (interdependencia, } \\
\text { globalización) }\end{array}$ & $\begin{array}{l}\text { - Transformaciones y desequilibrios en el mundo actual. } \\
\text { Interdependencia y globalización } \\
\bullet \text { El análisis de la globalización } \\
\text { • La globalización y los problemas del desarrollo }\end{array}$ \\
\hline $\begin{array}{c}\mathrm{PP} \\
\text { (principio de precaución) }\end{array}$ & $\begin{array}{l}\text { - La necesidad de búsqueda y aplicación de soluciones, sujetas al } \\
\text { principio de precaución } \\
\text { - Importancia de la aplicación del principio de precaución }\end{array}$ \\
\hline $\begin{array}{c}\mathrm{ET} \\
\text { (ética) }\end{array}$ & $\begin{array}{l}\text { - Desde este nuevo punto de vista ético es posible abordar el análisis de } \\
\text { determinados problemas característicos de la sociedad actual, (...) El } \\
\text { concepto de ciudadanía global, el desarrollo humano sostenible }\end{array}$ \\
\hline $\begin{array}{c}\text { ECO } \\
\text { (economía) }\end{array}$ & $\begin{array}{l}\text { - Describir algún caso que muestre las consecuencias medioambientales } \\
\text { de las actividades económicas }\end{array}$ \\
\hline
\end{tabular}


Desarrollo científico-tecnológico (DCT)

Aparecen 7 referencias a la actividad científico-tecnológica o tecnociencia. En 2 se subraya su aportación positiva a la sostenibilidad; en 3 se sugiere su responsabilidad en la génesis y también en la solución de la problemática; en 2 se expresa relación, pero sin concretar valoración.

\section{Problemas ambientales y sociales (PAS)}

Aparecen 34 referencias a la problemática ambiental y social, con diversos grados de concreción. Aunque la problemática ambiental es, más exactamente, socio-ambiental, el enunciado de los problemas suele enfatizar uno u otro aspecto. En el presente caso, 30 referencias pueden calificarse básicamente como "ambientales" y 4 como "sociales", en relación con la mencionada problemática.

En cuanto a las referencias a problemas ambientales, 7 de ellas son referencias genéricas, en las que se subraya la importancia de los problemas ambientales, la necesidad de implicarse en su solución, su escala local/global, etc. Otras 6 referencias corresponden al agua, su problemática y diversos aspectos relacionados con una gestión sostenible de este recurso. Hasta 5 referencias corresponden a la energía (problemática, ahorro, renovables y no renovables, etc.). Aparecen 4 referencias a la biodiversidad: valoración, pérdida, necesidad de mantenerla, etc.). 2 referencias se relacionan con los residuos (gestión, reciclaje) y 2 más corresponden a cambio climático. Finalmente, se recoge 1 referencia a vegetación; 1 a contaminación sin fronteras y 1 al agotamiento de los recursos.

4 referencias corresponden a aspectos sociales -problemática social- relacionados con la sostenibilidad: desigualdades; desplazamientos de la población; consumo y alimentación, respectivamente.

Entre las ausencias, cabe destacar, por ejemplo, la de referencias al territorio y su ordenación, a los problemas de erosión y desertificación o, en cuanto a aspectos sociales, al crecimiento demográfico y a los conflictos relacionados con el control de los recursos.

\section{Comportamientos, actitudes $(C)$}

Aparecen 11 referencias a actitudes, comportamientos, hábitos, etc. ya sean individuales o colectivos. En 7 de ellas se menciona la importancia de actuar, de intervenir en la toma de decisiones, los comportamientos, las actuaciones políticas, etc.; es decir, acción individual o colectiva. 2 se refieren a las actitudes favorables al desarrollo sostenible. 2 más subrayan la implicación de los hábitos en el medio ambiente y en el desarrollo sostenible.

Interdependencia, globalización (IG)

Aparecen 3 referencias a la interdependencia y/o a la globalización relacionadas con el campo semántico de la sostenibilidad.

Principio de precaución (PP)

Aparecen 2 referencias al principio de precaución

\section{Ética (ET)}

Aparece 1 referencia a la dimensión ética en relación con la sostenibilidad. 


\section{Economía (ECO)}

Aparece 1 referencia a la relación entre la actividad económica y sus efectos sobre el medio ambiente.

\section{DISCUSIÓN Y CONCLUSIONES}

En primer término conviene señalar que el discurso legislativo estudiado confiere una notable relevancia al concepto de sostenibilidad. Así, de un total de seis materias en cuyo currículo se incluye el concepto Desarrollo Sostenible, solamente dos son optativas, de lo que se deduce que es un concepto al que, en rasgos generales, y por parte de la administración educativa, se le da cierta relevancia en la última etapa educativa obligatoria. El currículum ofrece, por tanto, oportunidades para la educación para el desarrollo sostenible en la ESO, aunque las posibilidades reales seguramente dependen más de la voluntad y la capacidad de los centros y el profesorado que de un marco normativo, por lo demás insuficientemente explícito e incompleto en este aspecto.

En segundo lugar destacaríamos el hecho de que el discurso legislativo fomenta una idea de desarrollo sostenible propia del modelo dominante. Más allá de la visión que sostiene que "desarrollo" y "sostenible" son dos conceptos contradictorios e irreconciliables, con lo que "desarrollo sostenible" sería un oxímoron, se pueden contraponer dos modelos diferentes y contrapuestos (Huckle, 2005), derivados de una interpretación más o menos radical o profunda de su significado y alcance: por una parte el dominante, reformista, que se orienta a equilibrar el crecimiento económico, con consideraciones de protección ambiental y, en el mejor de los casos, con alguna preocupación por el bienestar social y, por otra, la visión radical, que propugna reformar la economía y la sociedad hacia formas que respeten los límites ecológicos y la justicia global. La visión dominante oculta la necesidad de que el desarrollo se produzca dentro de los límites ecológicos y fomenta una visión reduccionista y analítica, mientras que la crítica fomenta el pensamiento holístico o sistémico. El punto de vista radical del desarrollo sostenible propugna generar bienestar económico y justicia social considerando las restricciones de los sistemas de soporte vital del planeta. En esta visión, conceptos como equidad y suficiencia son de suma importancia, al igual que puntos de vista alternativos de la naturaleza, proponiendo una perspectiva biocéntrica frente a la perspectiva antropocéntrica dominante. El modelo radical parte de la premisa de que la civilización actual está entrando en una crisis terminal a causa del agotamiento de los recursos naturales, la contaminación creciente y la pérdida acelerada de biodiversidad. En suma, un conjunto de cambios ambientales afectados por la actividad humana, con especial referencia a cambios en los procesos que determinan el funcionamiento del sistema Tierra, lo que ha venido a denominarse cambio global (Duarte et al., 2006).

De acuerdo a esto, podemos afirmar que el discurso normativo analizado fomenta la visión dominante puesto que: a) Aborda de forma relevante el desarrollo sostenible desde el tecnocentrismo, enfatizando las posibilidades de la ciencia y la tecnología (tecnociencia) para la resolución de los problemas, básicamente los ambientales, aunque se aluda también a su responsabilidad en los mismos. Se presenta así la tecnología como la mejor herramienta para la protección del medio, sugiriendo implícitamente que la solución de la problemática ambiental es, fundamentalmente, una cuestión que compete 
a los expertos, y eludiendo de este modo la responsabilidad individual, social y política en su génesis y en sus posibles soluciones.

b) Enfatiza los aspectos ambientales frente a los sociales. Aspectos sociales tales como las desigualdades, las migraciones, el consumo, etc. de la problemática socio-ambiental resultan mucho más escasos -cuando no ausentes- en comparación con los más propiamente ambientales, tales como el agua, la energía, la biodiversidad, los residuos, etc. Cabe también señalar la ausencia de referencias a aspectos de tanta relevancia para la sostenibilidad como la ordenación del territorio, los problemas de erosión y desertificación, la demografía o los conflictos relacionados con el control de los recursos, entre otros.

c) Fomenta una visión de la realidad fragmentada, no sistémica. Son escasas referencias a la globalización y la interdependencia (solamente 3 referencias). En este mismo sentido, cabe señalar que las frecuentes referencias a determinados problemas ambientales (agua, energía, etc.) no se relacionan entre sí como parte y como consecuencia disfuncional de un determinado modelo socioeconómico.

d) Las escasas referencias a la dimensión ética de la crisis ambiental (1) y la relación entre economía y medio ambiente (1) -uno de los campos en que se desarrolla un debate más vivo y se generan perspectivas de análisis de mayor riqueza- sugieren un enfoque convencional y escasamente conflictivo del tratamiento de la sostenibilidad a nivel legislativo.

Siguiendo en esta línea argumental, del estudio realizado se deduce que los diversos conceptos asociados al campo semántico del desarrollo sostenible aparecen de forma desigual, destacando los más genéricos o consolidados en la bibliografía (desarrollo sostenible y sostenibilidad) y a continuación los asociados a la dimensión temporal (futuro sostenible) y al control (gestión sostenible). Además, en el discurso legislativo, la presencia de la sostenibilidad en las diversas materias resulta desigual, destacando en las Ciencias de la Naturaleza, Biología y Geología y Física y Química; y teniendo menor peso en asignaturas propias de las Ciencias Sociales, Geografía e Historia y Tecnología.

El enfoque de la sostenibilidad que se adopta en el Real Decreto 1631/2006 resulta, en líneas generales, superficial, fragmentado, desequilibrado y simplista. Ello sugiere la necesidad de replantear dicha normativa desde un compromiso radical hacia la grave problemática (social-económica-ambiental y cultural) que afronta actualmente la humanidad. Una necesidad que requiere además una contextualización en el período de crisis global económica y financiera en que nos hallamos desde 2008. La idea de sostenibilidad es central en un momento como el actual y lo es "porque emana de una muy clara toma de conciencia de la necesidad de reconstruir instituciones capaces de controlar la vida económica en nombre de los derechos de origen moral" (Touraine 2011: 123). Unos derechos, añadimos, complementarios de las obligaciones que tenemos hacia los demás y hacia la biósfera.

A la luz de los resultados obtenidos apuntamos la necesidad de que la normativa básica que regula el currículum en la enseñanza obligatoria y las diversas disposiciones que la desarrollan, deben tratar la sostenibilidad, un elemento de importancia crucial en la formación de la ciudadanía, a partir de un esquema inicial de referencia que integre las diversas dimensiones sociales y ambientales implicadas y asegure le presencia de éstas, de forma equilibrada en las diversas materias y mediante un tratamiento progresivo y pedagógicamente fundamentado a lo largo de esta etapa educativa. 


\section{AGRADECIMIENTOS}

Los co-autores de este trabajo son miembros del Grupo de Investigación de la Universidad de las Islas Baleares "Educación y Ciudadanía", que cuenta con la consideración de Grupo Competitivo y el patrocinio por parte de la Comunidad Autónoma de las Islas Baleares, a través de la Dirección General de Investigación, Desarrollo Tecnológico e Innovación de la Consejería de Innovación, Interior y Justicia, y la cofinanciación de fondos FEDER.

\section{REFERENCIAS BIBLIOGRÁFICAS}

Aragones, J. I., Izurieta, C., y Raposo, G. (2003). Revisando el concepto desarrollo sostenible en el discurso social. Psicothema, vol. 15, n. 2, 221-226.

Ärlemalm-Hagsér, E., y Sandberg, A. (2011). Sustainable development in early childhood education: In-service students' comprehension of the concept. Enviornmental Education Research, vol. 17, n. 2, 187-200.

Byrch, C., Kearins, K., Milne, M., y Morgan, R. (2007). Sustainable 'what'? A cognitive approach to understanding Sustainable Development. Qualitative Research in Accounting and Management, vol. 4, n. 1, 26-52.

Corney, G. (2006). Education for sustainable development: An empirical study of the tensions and challenges faced by geography student teachers. International Research in Geographical and Environmenatl Education, vol. 15, n. 3, 224-240.

Corney, G., y Reid, A. (2007). Student teachers'learning about subject matter and pedagogy in education for sustainable development. Environmental Education Research, vol. 13, n. 1, 33-54.

Cotton, D. R. E., Warren, M. F., Maiboroda, O., y Bailey I. (2007). Sustainable development, higher education and pedagogy: a study of lecturers' beliefs and attitudes. Environmental Education Research, vol. 13, n. 5, 579-597.

Duarte, C. (Coord.) (2006). Cambio global. Impacto de la actividad humana sobre el sistema Tierra. Madrid: CSIC.

Fergus, A. y Rowney, J. (2005). Sustainable development: lots meaning and oportunity. Journal of Bussiness ethics, vol. 60, n. 1, 17-27.

FINANCIAL TIMES. (1998, 7 de Enero). Natural Step to Sustainability. Financial Times.

Firth, R., Y Winter, C. (2007). Constructing education for sustainable development: The secondary school geography curriculum and inital teacher training. Environmental Education Research, vol. 13, n. $5,599-619$.

Huckle, J. (2005). Education for sustainable development. A briefing paper for the training and development agency for schools. Training and Development Agency for Schools. En línea; disponible en http://www.re-net.ac.uk/viewarticle2.aspx?contentId=11324 (12/10/2011).

Jóhannesson et al. (2011). Curriculum analysis and education for sustainable development in Iceland. Environmental education research, vol. 17, n. 3, 375-391.

Kemp, P. Y Martens, P. (2007). Sustainable development: how to manage something that is subjetive and never can be achieved? Sustainability: Science, practice and policy, vol. 3, n. 2, 1-10.

Mcgarr, O. (2010). Education for Sustainable Development in technology education in Irish schools: A curriculum analysis. International Journal of Technology and Design Education, vol. 20, n. 3, 317-332.

Moreira, S. S., y Carneiro, M. G. (2007). Las ideas de sostenibilidad de los alumnos de un curso de biología. Revista electrónica de enseñanza de las ciencias, vol. $6, n$. 1. En línea; disponible en http://reec.uvigo.es/volumenes/volumen6/ART13_Vol6_N1.pdf (12/10/2011). 
Reid, A., Petocz, P., y Taylor, P. (2009). Business students' conceptions of sustainability. Sustainability, vol. $1, n$ 3, 662-673.

Rheinländer, K. y Kramer, M. (2003). Green curricula? An analysis of environmentally oriented curricula in economics and business administration at German institutions of higher education. International Journal of Environment and Sustainable Development, vol. 2, n. 4, 391-411.

Rodríguez, M., Kohen, R., y Delval, J. (2008). El desarrollo sostenible en la mente del niño y el adolescente: El puente entre la naturaleza y la economía. Medio Ambiente y Comportamiento Humano, vol. 9, n. 1-2, 197-221.

Summers, M., Corney, G., y Childs, A. (2004). Student teachers' conceptions of sustainable development: The strating-points of geographers and scientists. Educational Research, vol. 46, n. 2, 163-182.

Tilbury, D. y Wortman, D. (2004). Engaging People in Sustainability, Commission on Education and Communication IUCN-the World Conservation Union. Cambridge: IUCN Publications Services Unit.

Touraine, A. (2011). Después de la crisis. Barcelona: Paidós.

UNESCO (2009). UNESCO: Conferencia mundial sobre Educación para el Desarrollo Sostenible, 2009: El Decenio de la Naciones Unidas de la Educación para un desarrollo durable. En línea, disponible en http://www.esd-world-conference-2009.org/es/informacion-de-base/deds. html (12/10/2011).

Vare, P. y Scott, W. (2007). Learning for a change: Exploring the relationship between education and sustainable development. Journal of Education for Sustainable Development, vol. 1, 191-198.

Walshe, N. (2008). Understanding students' conceptions of sustainability. Enviornmental Education Research, vol. 14, n. 5, 537-558.

Winter, C., y Firth, R. (2007). Knowledge about education for sustainable development: Four case studies of student teachers in english secondary schools. Journal of Education for Teaching, vol. 33, n. 3, 341-358. 
